\title{
Contrast-free MRI Protocol for Pituitary Assessment in Children with Growth or Puberty Disorders: A Practical Approach.
}

\author{
Marta Michali-Stolarska \\ Wrocław Medical University \\ Andrzej Tukiendorf \\ Wrocław Medical University \\ Wrocław Medical University \\ Anna Zacharzewska-Gondek \\ Wrocław Medical University \\ Joanna Chrzanowska \\ Wrocław Medical University \\ Joanna Bladowska \\ Wrocław Medical University
}

Jagoda Jacków-Nowicka ( $\square$ jagoda.jackow-nowicka@umed.wroc.pl )

\section{Research Article}

Keywords: Magnetic Resonance Imaging, Pituitary Gland, Child, Contrast Media, Gadolinium

Posted Date: March 10th, 2021

DOI: https://doi.org/10.21203/rs.3.rs-266238/v1

License: (c) (i) This work is licensed under a Creative Commons Attribution 4.0 International License.

Read Full License 
TITLE:

Contrast-free MRI protocol for pituitary assessment in children with growth or puberty disorders: a practical approach.

Marta Michali-Stolarskaa , MD; Andrzej Tukiendorfb, Prof.; Jagoda Jacków-Nowicka ${ }^{a^{*}}$, MD, PhD, Anna Zacharzewska-Gondeka, MD; Joanna Chrzanowskac, MD, PhD; Joanna Bladowska Prof.

\begin{abstract}
Affiliations:
a Department of General and Interventional Radiology and Neuroradiology, Wroclaw Medical University, Poland

b Department of Public Health, Wroclaw Medical University, Wroclaw, Poland

c Department of Developmental Endocrinology and Diabetology, Wroclaw Medical University, Wroclaw, Poland
\end{abstract}

\title{
Address correspondence to:
}

Jagoda Jacków-Nowicka,

Department of General and Interventional Radiology and Neuroradiology,

Wroclaw Medical University, Wroclaw, Poland

Borowska 213 Str.,

50-556 Wroclaw, Poland,

Tel. 0048 (71) 733 1668, Fax. 0048 (71) 734 1669;

e-mail: jagoda.jackow-nowicka@umed.wroc.pl

Financial support statement: Wroclaw Medical University Grant SUB.C270.21.020. 


\section{ABSTRACT \\ Objectives}

Most of the pituitary MRI examinations in children with growth or puberty disorders (GPD) might not require gadolinium-based contrast agent (GBCA) administration.

Methods

Retrospective re-analysis of contrast-enhanced 567 pituitary MRIs of children with GPD. Two sets of sequences were created from each MRI examination: Set1 - common sequences without contrast administration and Set2 - common pre- and post-contrast sequences. The differences in the visibility of pituitary lesions between sets were statistically analyzed.

Results

The overall frequency of Rathke's cleft cysts was $11.6 \%$, ectopic posterior pituitary $3.5 \%$ and microadenomas $0.9 \%$. Lesions visible without contrast administration accounted for $85 \%$ of cases, while lesions diagnosed only after contrast injection accounted for $0.18 \%$ of all patients. Statistical analysis showed the advantage of antero-posterior (AP) pituitary dimension over other criteria in determining the appropriateness of using contrast in pituitary MRIs. The AP dimension was the most significant factor in logistic regression analysis: $\mathrm{OR}=2.23,95 \% \mathrm{CI}, 1.35-3.71$, $\mathrm{p}$ value $=0.002$ and in ROC analysis: AUC: $72.9 \%$ with cut-off value $7.5 \mathrm{~mm}$, with sensitivity/ specificity rates: $69.2 \% / 73.5 \%$.

\section{Conclusions}

In most cases, the use of GBCA in pituitary MRI in children with GPD is unnecessary. The additional advantages of GBCA omission include: shortening the time of MRI examination and of general anesthesia. 


\section{KEY WORDS}

Magnetic Resonance Imaging; Pituitary Gland; Child; Contrast Media, Gadolinium

\section{KEY POINTS}

- In most cases, non-contrast MRI of pituitary gland in children with growth or puberty disorders is sufficient for correct gland assessment and further clinical management.

- The antero-posterior pituitary dimension (the cut-off value $=7.5 \mathrm{~mm}$ ) was the most significant factor in determining the appropriateness of using gadolinium-based contrast agent.

- A practical approach to MRI assessment of the pituitary gland in children with growth or puberty disorders will optimize the management of patients before hormone therapy.

\section{ABBREVIATIONS}

- AUC - Area Under Curve

- AP - antero-posterior dimension

- BW - Body weight

- CC - cranio-caudal dimension

- children with GPD - children with growth or puberty disorders

- CI95\% - Confidence 95\% intervals

- EPP - ectopic posterior pituitary

- GBCA - gadolinium-based contrast agent 
- MA - Microadenoma

- MRI - Magnetic resonance imaging

- OR - Odds ratio

- PSIS - Pituitary Stalk Interruption Syndrome

- RCC - Rathke's cleft cyst

- ROC analysis - Receiver operating characteristic analysis

- Set1 - common pituitary MRI sequences without contrast administration

- Set2 - conventional pituitary MRI (which includes common pre- and post-contrast sequences)

- SP - signal pattern

- $\quad$ T1-WI and T2-WI - T1-weighted images and T2-weighted images

- TR - transverse dimension

- V - gland volume 


\section{INTRODUCTION}

One of the causes of hormone-related disorders in childhood physical development may be the presence of a lesion in the pituitary gland region. After endocrinological assessment, pituitary changes exclusion is one of the most important elements of diagnostic management of growth and puberty disorders (GPD) in children.[1], [2] The method of choice for pituitary assessment is magnetic resonance imaging (MRI).[2] Actually, the MRI protocol of the pituitary gland includes images before and after administration of gadolinium-based contrast agent (GBCA),[2], [3] which according to current knowledge is not neutral to the human body. Apart from the commonly known gadolinium-related side effects, such as allergic reactions as well as nephrogenic systemic fibrosis (in kidney failure),[4], [5] recently scientific literature alerts about the possible accumulation of gadolinium compounds in some brain structures (globus pallidus and dentate nucleus).[6] These observations are of concern to the medical community and the questions are self-evident: will the developing brains of children have an increased susceptibility to long-term, potentially neurotoxic effects of GBCA deposits[7], [8] and whether it is possible to limit the use of GBCA in pituitary MRI in children.[9], [10] Additionally, the omission of GBCA administration would shorten the examination time and the duration of general anesthesia, which is necessary in the youngest patients.[2]

Over the past decade, the concern about possible harmful effects of anesthetics and sedatives used in neonates and young children have increased.[11]-[14] Studies on animals demonstrate that repeated or prolonged administration of these drugs is potentially neurotoxic and may cause delayed cognitive development.[14]-[16] 
The aim of the study was to assess the diagnostic value of non-contrast MRI of the pituitary gland in children with GPD and to determine the criteria indicating the necessity to perform post-contrast examination.

We hypothesized that most of the pituitary MRI examinations in children with GPD do not require GBCA administration.

To the best of our knowledge, this is the first study focusing on performing MRI of the pituitary gland without gadolinium administration in children.

\section{MATERIALS AND METHODS}

\section{Study Participants}

For this retrospective study, after approval by the local ethics committee (opinion No. 117/2019), a total of 579 young patients from the Department of Developmental Endocrinology and Diabetology who met several inclusion and exclusion criteria were selected. Inclusion criteria were as follows: contrast-enhanced pituitary MRI examination carried out in our Radiology Department, age below 18 years, and growth or puberty disorders. Exclusion criteria were: inadequate MRI due to artifacts $(\mathrm{n}=1)$ or absence of contrast agent $(\mathrm{n}=11)$. Consecutive pituitary MRIs of 567 children between January 2007 and May 2020, were retrospectively reevaluated (Table 1).

For the purposes of statistical analysis, two sets of sequences (Figure 1) were created from each pituitary MRI examination of every patient: Set1 including common sequences without contrast administration and Set 2 which included common pre- and post-contrast sequences (conventional MRI examination). Set1 was the reference ("control") group for Set2 in each patient. Each pair of sets (Set1 and Set2) from each patient was assessed separately and independently, and the evaluating neuroradiologist was blinded to patient information. 
The patients were deliberately not divided into age groups, because they were individuals with hormonal disorders and in their case, according to our observations (the head of the project has 20 years of experience in pituitary imaging) and reports of other research centers,[17] the dimensions of the pituitary gland are not directly proportional to age, which is in a healthy population. 


\section{Technical Details and Image Analysis}

The MR examinations were carried out using two devices: GE 1.5T MR Signa HDx $(n=425)$ and $3 T$ Philips Ingenia ( $\mathrm{n}=142)$, using 16-channel coils dedicated to the head and neck area.

T1 weighted images (T1-WI) and T2 weighted images (T2-WI) were taken in the coronal and sagittal planes using thin $3 \mathrm{~mm}$ slices before and after intravenous administration of the macrocyclic GBCA. The contrast dose was $0.1 \mathrm{mmol} / \mathrm{kg}$ body weight $(0.2 \mathrm{ml} / \mathrm{kg} \mathrm{BW})$. General anesthesia was additionally used in younger patients (below age of 7 years) to exclude movement artifacts.

The size (gland volume and three dimensions) and the morphology of the pituitary gland, the possible presence and then location, signal pattern (SP) and potential occurrence of contrast enhancement of focal lesions were retrospectively reassessed on the GE ADW 4.6 as well as the Philips IntelliSpace workstations.

\section{Statistical analysis}

The computation was performed in the R statistical platform.[18] The receiver operating characteristic (ROC) curve analysis was performed using the "pROC” R package.[19]

According to the statistical analysis of the differences in the visibility of focal lesions between Set1 (common pituitary MRI sequences without contrast administration) and Set2 (conventional MRI examination with common pre- and post-contrast sequences), we were able to assess the rightness of using GBCA in children with GPD.

First, differences in the diagnoses of pituitary focal lesions in Set 1 and Set 2 were determined. The analysis covered such risk factors as age, sex, puberty, pituitary geometrical dimensions - including antero-posterior (AP), transverse (TR), cranio-caudal dimension (CC), gland volume, and signal 
pattern (SP) of the identified focal lesions. The risk for differences in these clinical diagnoses was statistically evaluated using logistic regression and expressed by the classic odds ratio (OR) based on the gathered clinical material.

The cut-offs for the presumed risk factors were estimated using ROC analysis, to distinguish between the negative effect (which is the unnecessary use of GBCA) and positive effect (correct GBCA administration) of the post-contrast examination in conventional pituitary MRI.

\section{RESULTS}

\section{Patient Characteristics}

Patient characteristics are shown in Table 1. A total of 567 children (boys/girls, 308/259; mean age \pm standard deviation (SD), $6.99 \pm 2.59$ years; range, $0.9-17.4$ years), including short stature $n=509$ (boys/girls, 294/215), gigantism $n=5(3 / 2)$, precocious puberty $n=54(11 / 43)$ were retrospectively reevaluated.

\section{The frequency of focal lesions}

In our study, Rathke's cleft cyst (RCC) appeared in 66 patients (11.6\%), ectopic posterior pituitary (EPP) was visible in 20 patients (3.5\%) with clinically confirmed Pituitary Stalk Interruption Syndrome (PSIS) and microadenoma (MA) was present in 5 children (0.9\%), additionally in Sets 1 (sequences without GBCA administration) they were already visible in 53/20/4 cases (RCC/EPP/ MA) respectively, that is, in about $80 \%$ of cases in both RCC and MA and $100 \%$ of EPP. When assessing the pituitary gland for the presence of a tumor, in Sets2 (conventional pituitary MRI which included sequences after GBCA administration) compared to Sets 1 the result has changed significantly in only $0,18 \%$ of all patients providing the diagnosis of 1 MA which was not visible on non-contrast MRI (Set1). Moreover, Sets2 also showed 13 new benign RCCs.

\section{Pituitary dimensions}


Logistic regression revealed that only the AP dimension of the pituitary gland had statistically significant $(\mathrm{P}<0.05)$ chances for diagnostic incorrectness, with the following results: $\mathrm{OR}=2.23$, $95 \% \mathrm{CI}, 1.35$ to 3.71 , $\mathrm{p}$-value $=0.002$ (Table 2 ), while the $\mathrm{CC}$ dimension and the gland volume were on the border of statistical significance $(\mathrm{P}<0.1)$.

The forest plot (Figure 2) based on the logistic regression analysis illustrates the advantage of the AP dimension, which, unlike other factors, does not cross the line of no difference (1.0 value), and is therefore statistically significant.

From the ROC analysis shown in Figure 3, with the following results: AUC:72.9\% (58.0\%-87.8\%), cut-off value $7.5 \mathrm{~mm}$, sensitivity/specificity rates: $69.2 \% / 73.5 \%$, it can be established that the AP dimension is a statistically significant (AUC lower CI 95\% >50\%) predictor of the appropriateness of contrast administration in the diagnosis of pituitary focal lesions in children with GPD. Classification of patients based on the AP cut-off $=7.5 \mathrm{~mm}$ indicates the proper post-contrast diagnostics in $(\mathrm{AUC} \sim 73 \%=) 3$ out of 4 patients. What is more, ROC analysis of the AP dimension shows the best fitted combination of sensitivity (69\%) and specificity (74\%) rates.

The statistical interpretation of the ROC analyses shown in Figures 4 and 5 is analogous. Both the CC dimension and the gland Volume according to the ROC analysis are statistically significant predictors in proper GBCA application in the diagnostic management of the discussed group of children. However, classification of patients based on the CC/Volume cut-off values $=4.5 \mathrm{~mm} / 123$ $\mathrm{mm} 3$ (respectively) indicate a correct post-contrast diagnosis less frequently compared to the AP dimension, reaching AUC $\sim 66 \% / 69 \%$ for CC/Volume (respectively). The ROC curves, together with supplementary statistical estimates, are displayed graphically in Figures 3-5.

Signal intensity of the lesion 
Statistical analysis showed that the signal pattern (SP) of pituitary lesion in a prediction of accurate contrast administration in the diagnosis of children with GPD is not statistically significant ( $p>0.05)$. Furthermore, our observations showed that the SPs of such lesions are varied. 


\section{DISCUSSION}

Focal lesions of the pituitary gland in the population of healthy children are unusual, and although in children with hormonal disorders this is also a rare phenomenon, it is more common than in healthy individuals.[1]

Studies on pituitary changes in the pediatric population were for a long time unavailable. Only in 2019 did Souteiro et al. in a study on children with almost 16000 patients examine the occurrence of all pituitary abnormalities with an incidence rate of 257/100,000 patients.[20]

The results of these authors indicated that the pathology frequency was as follows: RCC in $0.03 \%$, EPP in $0.02 \%$ and adenomas in $0.04 \%$ of all patients.

In our study of 567 pediatric patients with GPD, three types of focal pituitary lesions were found: RCC, EPP and MA $(11.6 \%, 3,5 \%$ and $0.9 \%$ of all patients, respectively). The frequency of focal lesions in our patient group is significantly higher than in the cited study on generally healthy children, most likely due to the characteristics of both pediatric populations.[20] Güneş et al. had similar observations of RCC in children with endocrine disorders.[2]

Moreover, our observations showed that in common pituitary MRI sequences without contrast administration (Set1), focal lesions were already visible in about $85 \%$ of cases.

In conventional pituitary MRIs, which included common pre- and post-contrast sequences (Sets2), compared to Sets 1 the outcome of pituitary MRI assessment changed only in $2.5 \%$ of all patients (mostly benign RCCs) and, importantly, only one patient $(0.18 \%)$ had MA.

The appearance of both RCC, EPP and MA are almost pathognomonic, thanks to their characteristic location. RCCs are present between the anterior and posterior lobe[21], EPP most often located on the floor of 3rd ventricle, at the median eminence [10] and MAs situated within the anterior lobe. 
It is well known that RCC is a benign lesion,[23] and if it is small and does not cause a mass effect, it should not be of concern to radiologists or clinicians. Furthermore, its location is so distinctive that the GBCA administration in this case is completely unnecessary.

So called "bright spot" visible on T1-weighted non-contrast MR images in the floor of the third ventricle enables identification of EPP without post-contrast examination.[10]

The presence of a focal lesion in the anterior pituitary lobe, which corresponds to MA[22] would seemingly indicate the need for gadolinium administration. However, in light of recent studies incidentalomas should not be considered a contraindication to hormone therapy.[1] Consequently, the identification of MA-like tumors in a non-contrast examination should not change clinical management of pediatric patients with hormonal disorders, therefore the diagnosis of MA does not need to be confirmed by a post-contrast MRI. Following this lead, one can come to interesting conclusions: even in the case of an incidentaloma not visible in the non-contrast examination, its potential detection after GBCA administration does not affect the clinical management of children with hormonal disorders. Of course, we should not generalize and the profit and loss account should be considered on a case-by-case basis.

As mentioned above, the SP of EPP is very characteristic[10], while SPs of RCC and MA depend on their content.[24], [25] What is more, as our study found, the latter can be very diverse. In addition, our statistical analysis showed that the SP of pituitary focal lesion is not statistically significant as a predictive factor of the accuracy of contrast administration in the diagnostics of children with GPD and thus does not affect the results of post-contrast examination.

This fact, combined with typical RCC, EPP or MA locations and redundant MA-like tumors confirmation with GBCA, it seems that after excluding the presence of a pituitary lesion the only 
factor influencing the decision to administer GBCA (that is, to perform a conventional pituitary MRI examination) in the pediatric population is the size of the pituitary gland.

The appropriateness of using contrast in pituitary MRIs in children with GPD can be measured by geometric factors, the advantage of which was demonstrated by statistical analysis (Table 2,3 and Figures 2-5). The ROC analysis showed that the CC dimension and gland volume are statistically significant, and logistic regression defined them as borderline values.

The AP dimension is the most accurate factor as a statistically significant predictor in two independent analyzes. Moreover, the ROC analysis itself also indicates the advantage of the AP dimension over other criteria, as it shows the best fitted combination of sensitivity and specificity rates, which indicate the high ability of the predictor to use the GBCA correctly when required, and the proper omission of GBCA administration when unnecessary.

Another argument for the superiority of the AP dimension over others is the fact that children with precocious puberty usually have an enlarged pituitary gland with a convex upper outline, which automatically increases the $\mathrm{CC}$ dimension, which obviously is not related to the presence of a focal lesion of this gland.[26], [27]

Combining the results of our statistical analysis with the above-mentioned literature data, we believe that in the practical approach only the AP dimension should be taken into account when assessing the size of the pituitary gland.

We hypothesized that administration of GBCA in pituitary MRI is unnecessary in the diagnostics of children with GPD. According to the ROC analysis (Fig. 3), the hypothesis holds true for the small size of the pituitary gland. With the AP dimension above the cut-off value $(>7.5 \mathrm{~mm})$, this hypothesis becomes false and GBCA should be administered in such cases. 
According to the ROC analysis, $73 \%$ of patients with an AP dimension $>7.5 \mathrm{~mm}$ should receive GBCA, and although the remaining $27 \%$ of patients in this group will receive GBCA unnecessarily, statistical analysis showed that the benefit of conventional examination (with pre- and post-contrast sequences) exceeds the potential losses and it is certainly a better solution than using GBCA for all individuals without exceptions.

In children with GPD, a pituitary-oriented MRI is performed prior to hormonal treatment in order to exclude organic causes of hormonal disorders. The conventional MRI examination protocol includes intravenous administration of GBCA, and in younger patients, additionally sedation is used to avoid motor artifacts.

Gadolinium deposition has been a very popular topic in recent years and the current state of knowledge is changing dynamically. The literature is consistent with the occurrence of the accumulation phenomenon of linear GBCA after their (especially) repeated administration[28], e.g. in the deep structures of the brain.[6] Opinions, however, are divided on the potential accumulation of macrocyclic contrasts, which are commonly considered a safer option and therefore often dedicated to pediatric examinations.[5], [29]-[31] Another aspect is the potential association between gadolinium depositions and possible clinical consequences, which has not been confirmed so far,[31], [32] but further observations of the long-term effects of this matter need to be carried out, especially in children due to the sensitivity of their developing brain and life span.[7], [8] At present, no histological changes have been confirmed that would arise as a result of gadolinium deposition in the brain tissue after its multiple administration, however, larger studies were conducted only on the adult population,[6], [33], [34] and the study of children was performed on a small number of patients.[8] 
However, the clinical significance and potential long-term consequences of the administration (especially repeated) of gadolinium even in individuals with normal renal function still remain unclear.[5], [32] This is evidenced by the position of the European Medicines Agency, which recommends using the lowest sufficient dose of macrocyclic GBCA and only in cases where native scans are insufficient[33] and the European Society of Urogenital Radiology, which recommends avoiding unnecessary administration of GBCA in children, especially in newborns and infants.[35] The U.S. Food and Drug Administration is also interested in this phenomenon and continuing to assess its effects in the human body.[36]

In our opinion, GBCA administration should be omitted in most pituitary MRIs in children with GPD. Therefore we propose the practical approach for pituitary MRI assessment (Figure 6). Worldwide, similar trends are also emerging in other fields of medicine where, especially in studies on the pediatric population scientists are trying to abandon GBCA administration[4], [37], [38] or at least reduce its dose.[9], [10]

There are also few studies in the literature indicating a need to reconsider the use of GBCA in pituitary MRI, which is not always necessary for the correct interpretation of the sellar region.[38], [39]

As with the harmfulness of gadolinium deposits, scientists are also divided over the toxicity of anesthetics in children[11]-[14] Animal studies have confirmed the neurotoxicity of anesthetics and sedatives on the developing brain tissue,[15], [16] and while these observations cannot be directly extrapolated to the pediatric population, they are of concern. Due to the specificity of the studied age group, most research are retrospective, and therefore not ideal. Some of these studies have found an association between exposure to anesthetics and neurobehavioral problems or cognitive impairment in the later stages of development, which is especially noticeable with repeated or prolonged exposure.[12], [13] 
Although several new studies have shown no noticeable neurocognitive impairment in children after short-term anesthetics exposure,[12], [14] however, as with the gadolinium deposition, the long-term side effects of such exposure are not yet known.

Due to constant concerns about the potential harmfulness of GBCA and the potential risk of general anesthesia in younger children, and in accordance with the results presented above, in our opinion, GBCA administration in unquestionable cases is not only unnecessary, but even could be considered an impropriety in the medical decision-making process.

Out of concern for the welfare of the youngest patients, we designed the procedure algorithm (Figure 6) for pituitary MRI assessment in children with GPD before hormonal therapy. The suggested MR protocol includes only T1 and T2-WI in the coronal and sagittal planes without contrast administration. Post-contrast examination may be omitted if the native MR examination meets the following criteria:

1. The pituitary gland must not present any focal lesions on $\mathrm{T} 1 \mathrm{and} /$ or $\mathrm{T} 2$ image or the lesion must be in the typical location for RCC, EPP or MA.

2. The AP dimension of the pituitary gland must be smaller than the cut-off value $(7.5 \mathrm{~mm})$.

Obviously, if a suspicious or potentially malignant lesion outside the sellar region is detected, not only should GBCA be administered, but the scope of the examination should also be extended to include a complete MRI protocol of the brain.

The present study has several limitations. First, it has a retrospective design; second, none of our patients underwent surgery, therefore histopathological confirmation of the diagnoses made by MRI was not possible. Additionally, the subgroups of children aged $<2$ and $>12$ years were small. 
Despite its limitations, to the best of our knowledge this is the first study involving an attempt of complete GBCA omission in pituitary MRI in children.

\section{CONCLUSION}

The results of our research have relevant implications for clinical practice or health policy.

For most children, it will be possible to shorten the examination time by omitting GBCA administration, which in turn has a number of advantages. Briefer examination means reduced time of general anesthesia in younger children.

Furthermore, reducing the procedure time would increase the chances of obtaining good-quality MRI of the pituitary gland in a conscious young patient who is, however, old enough not to move during the shortened examination without GBCA administration.

Shortening the examination time is also important for health policy due to its economic aspects. Reducing the number of post-contrast examinations will save time, during which more children with GPD waiting in line might be examined. This aspect is particularly important in countries where access to MRI examinations is limited. This will increase the availability of MRI examinations for waiting children and accelerate their further clinical management.

\section{REFERENCES}

[1] Derrick, K. M., Gomes, W. A., Gensure, R. C. Incidence and Outcomes of Pituitary Microadenomas in Children with Short Stature/Growth Hormone Deficiency. Horm. Res. Paediatr. vol. 90, no. 3, pp. 151-160 (2018).

[2] Güneş, A., Özbal Güneş, S. The neuroimaging features of rathke's cleft cysts in children with endocrine-related diseases. Diagnostic Interv. Radiol. vol. 26, no. 1, pp. 61-67 (2020).

[3] Karimian-Jazi, K. Pituitary gland tumors. Radiologe. vol. 59, no. 11, pp. 982-991 (2019).

[4] Holowka, S., Shroff, M., Chavhan, G. B. Use and Safety of Gadolinium Based Contrast 
Agents in Pediatric MR Imaging. Indian J. Pediatr. vol. 86, no. 10, pp. 961-966 (2019).

[5] McDonald, R. J. et al. Gadolinium retention: A research roadmap from the $2018 \mathrm{NIH} / \mathrm{ACR} /$ RSNA workshop on gadolinium chelates. Radiology. vol. 289, no. 2, pp. 517-534 (2018).

[6] Kanda, T. et al. Gadolinium-based contrast agent accumulates in the brain even in subjects without severe renal dysfunction: Evaluation of autopsy brain specimens with inductively coupled plasma mass spectroscopy. Radiology. vol. 276, no. 1, pp. 228-232 (2015).

[7] Elbeshlawi, I., AbdelBaki, M. S. Safety of Gadolinium Administration in Children. Pediatr. Neurol. vol. 86, pp. 27-32 (2018).

[8] McDonald, J. S. et al. Intracranial gadolinium deposition following gadodiamide-enhanced magnetic resonance imaging in pediatric patients: A case-control study. JAMA Pediatr. vol. 171, no. 7, pp. 705-707 (2017).

[9] Portocarrero-Ortiz, L., Bonifacio-Delgadillo, D., Sotomayor-González, A., Garcia-Marquez, A., Lopez-Serna, R. A modified protocol using half-dose gadolinium in dynamic 3-Tesla magnetic resonance imaging for detection of ACTH-secreting pituitary tumors. Pituitary. vol. 13, no. 3, pp. 230-235 (2010).

[10] Rennert, J., Doerfler, A. Imaging of sellar and parasellar lesions. Clin. Neurol. Neurosurg. vol. 109, no. 2, pp. 111-124 (2007).

[11] O'Leary, J. D. Human Studies of Anesthesia-Related Neurotoxicity in Children: A Narrative Review of Recent Additions to the Clinical Literature. Clin. Perinatol. vol. 46, no. 4, pp. 637-645 (2019).

[12] Warner, K. S., Zaccariello, D. O. Neuropsychological and Behavioral Outcomes after Exposure of Young Children to Procedures Requiring General Anesthesia, The Mayo Anesthesia Safety in Kids (MASK) Study. Anesthesiology. no. 129(1), pp. 89-105 ( 2018).

[13] Bjur, K. A., Payne, E. T., Nemergut, M. E., Flick, D. Hu, \& R. P. Anesthetic-Related Neurotoxicity and Neuroimaging in Children: A Call for Conversation. J. Child Neurol. vol. 32, no. 6, pp. 594-602 (2017).

[14] Sun, L. S. et al. Association between a single general anesthesia exposure before age 36 months and neurocognitive outcomes in later childhood. JAMA - J. Am. Med. Assoc. vol. 315, no. 21, pp. 2312-2320 (2016).

[15] Barton, K., Nickerson, J. P., Higgins, T., Williams, R. K. Pediatric anesthesia and neurotoxicity: what the radiologist needs to know. Pediatr. Radiol. vol. 48, no. 1, pp. 31-36 (2018).

[16] Brambrink, A. M. et al. Isoflurane-induced neuroapoptosis in the neonatal rhesus macaque brain. Anesthesiology. vol. 112, no. 4, pp. 834-841 (2010).

[17] Dumrongpisutikul, N., Chuajak, A., Lerdlum, S. Pituitary height at magnetic resonance imaging in pediatric isolated growth hormone deficiency. Pediatr. Radiol. vol. 48, no. 5, pp. 694-700 (2018).

[18] R Team Core, R: A Language and Environment for Statistical Computing Version 4.0.2. R. Foundation for Statistical Computing Vienna, Austria (2020).

[19] Robin, X. et al. pROC: An open-source package for R and S+ to analyze and compare ROC curves, BMC Bioinformatics. vol. 12 (2011).

[20] Souteiro, P. et al. Pituitary incidentalomas in paediatric age are different from those described in adulthood. Pituitary. vol. 22, no. 2, pp. 124-128 (2019).

[21] Takanashi, J. I., Tada, H., Barkovich, A. J., Saeki, N., Kohno,Y. Pituitary cysts in childhood evaluated by MR imaging. Am. J. Neuroradiol. vol. 26, no. 8, pp. 2144-2147 (2005).

[22] Giannattasio, G., Bassetti, M., Human pituitary adenomas. Recent advances in morphological 
studies. J. Endocrinol. Investig. Off. J. Ital. Soc. Endocrinol. vol. 13, no. 5, pp. 435-454 (1990).

[23] Teramoto, A., Hirakawa, K., Sanno, N., Osamura, Y. Incidental pituitary lesions in 1,000 unselected autopsy specimens. Radiology. vol. 193, no. 1, pp. 161-164 (1994).

[24] Sumida, M. K. et al. Rathke cleft cysts: correlation of enhanced MR and surgical findings. AJNR Am J Neuroradiol. vol. 15, no. 3, pp. 525-32 (1994).

[25] Guaraldi, F., Storr, H. L., Ghizzoni, L., Ghigo, E., Savage, M. O. Paediatric pituitary adenomas: A decade of change. Horm. Res. Paediatr. vol. 81, no. 3, pp. 145-155 (2014).

[26] Kao, S. C. S., Cook, J. S., Hansen, J. R., Simonson, T. M. MR imaging of the pituitary gland in central precocious puberty, Pediatr. Radiol. vol. 22, no. 7, pp. 481-484 (1992).

[27] Sharafuddin, G. E. R., Luisiri, M J, Garibaldi, A., Fulk, L. R., Klein, D. L., Gillespie J. B., MR imaging diagnosis of central precocious puberty: importance of changes in the shape and size of the pituitary gland. AJR Am J Roentgenol. vol. 162, pp. 1167-1173 (1994).

[28] Quattrocchi, C. C. et al. Effect of Age on High T1 Signal Intensity of the Dentate Nucleus and Globus Pallidus in a Large Population Exposed to Gadodiamide, Invest. Radiol. vol. 53, no. 4, pp. 214-222 (2018).

[29] Kanda, T. et al. High signal intensity in dentate nucleus on unenhanced T1-weighted MR images: Association with linear versus macrocyclic gadolinium chelate administration. Radiology. vol. 275, no. 3, pp. 803-809 (2015).

[30] Rossi Espagnet, M. C., Bernardi, B., Pasquini, L., Figà-Talamanca, L., Tomà, P., Napolitano, A. Signal intensity at unenhanced T1-weighted magnetic resonance in the globus pallidus and dentate nucleus after serial administrations of a macrocyclic gadolinium-based contrast agent in children. Pediatr. Radiol. vol. 47, no. 10, pp. 1-8 (2017).

[31] Gulani,V., Calamante, F., Shellock, F. G., Kanal, E., Reeder, S. B. Gadolinium deposition in the brain: summary of evidence and recommendations. Lancet Neurol. vol. 16, no. 7, pp. 564-570 (2017).

[32] Mallio, C. A., Rovira, À., Parizel, P. M., Quattrocchi, C. C. Exposure to gadolinium and neurotoxicity: current status of preclinical and clinical studies. Neuroradiology. vol. 62, no. 8, pp. 925-934 (2020).

[33] EMA's final opinion confirms restrictions on use of linear gadolinium agents in body scans. Eur. Med. Agency. (2017).

[34] Fingerhut, S. et al. Gadolinium-based contrast agents induce gadolinium deposits in cerebral vessel walls, while the neuropil is not affected: An autopsy study. Acta Neuropathol. vol. 136, no. 1, pp. 127-138 (2018).

[35] Thomsen, H. S. et al. Nephrogenic systemic fibrosis and gadolinium-based contrast media: Updated ESUR Contrast Medium Safety Committee guidelines. Eur. Radiol. vol. 23, no. 2, pp. 307-318 (2013).

[36] FDA, FDA Drug Safety Communication: FDA warns that gadolinium-based contrast agents (GBCAs) are retained in the body; requires new class warnings. (2018).

[37] Dünger, D., Krause, M., Gräfe, D., Merkenschlager, A., Roth, C., Sorge, I. Do we need gadolinium-based contrast medium for brain magnetic resonance imaging in children? Pediatr. Radiol. vol. 48, no. 6, pp. 858-864 (2018).

[38] Bonneville, J. F. A plea for the T2W MR sequence for pituitary imaging. Pituitary. vol. 22, no. 2, pp. 195-197 (2019).

[39] Bonneville, J. F. Long term MRI surveillance of pituitary macroadenomas: gadolinium is not obligatory. Pituitary. vol. 22, no. 1, pp. 100-102 (2019). 
TABLES:

Table 1: Clinical Characteristics of the Patients

Data are numbers of patients, divided by gender in parentheses: boys/girls.

* Data are means \pm standard deviations.

\begin{tabular}{|l|l|} 
Characteristic & \multicolumn{1}{l}{ Value } \\
\hline No. of patients & 567 \\
\hline Age $(\mathbf{y})^{*}$ & $6.99 \pm 2.59$ \\
\hline Range & $0.9-17.4$ \\
\hline No. of male patients & 294 \\
\hline No. of female patients & 259 \\
\hline clinical diagnosis & \\
\hline \multicolumn{1}{|c|}{ short stature } & $509(294 / 215)$ \\
\hline gigantism & $5(3 / 2)$ \\
\hline precocious puberty & $54(11 / 43)$ \\
\hline
\end{tabular}

Table 2: Odds ratios (ORs) of lack of correct diagnosis of pituitary lesions in a conventional MRI examination (which includes common pre- and post-contrast sequences).

Odds ratios (ORs) with confidence 95\% intervals (CI95\%) and p-values. Table includes tree pituitary dimensions (antero-posterior [AP], cranio-caudal [CC], transverse [TR]) and gland volume [V], where AP is statistically significant $(\mathrm{P}<0.05), \mathrm{CC}$ and $\mathrm{V}$ are on the border of statistical significance $(\mathrm{P}<0.1)$ and TR is statistically insignificant.

\begin{tabular}{|c|c|c|c|}
\hline Risk factor & OR & CI95\% & p-value \\
\hline $\mathbf{A P}[\mathbf{m m}]$ & 2.23 & $(1.35,3.71)$ & .002 \\
\hline $\mathrm{CC}[\mathrm{mm}]$ & 1.40 & $(0.97,2.00)$ & .071 \\
\hline $\mathbf{V}\left[\mathbf{m m}^{3}\right]$ & 1.005 & $(0.999,1.011)$ & .055 \\
\hline TR [mm] & 0.93 & $(0.64,1.34)$ & .679 \\
\hline
\end{tabular}


Table 3: Summary of the results of the Receiver Operating Characteristic (ROC) analysis.

Data in parentheses are $95 \%$ CIs. P values are for comparisons of the area under the receiver operating characteristic curve (AUC). Classifications of patients based on calculated Cut-offs, which indicate the proper diagnosis in a conventional pituitary MRI examination (which includes common pre- and post-contrast sequences).

Table includes two pituitary dimensions (antero-posterior [AP], cranio-caudal [CC]) and gland volume $[\mathrm{V}]$. All factors included in the table are statistically significant according to the ROC analysis (AUC lower CI 95\% >50\%), but only AP dimension is statistically significant according to the logistic regression analysis $(\mathrm{P}=.002)$ and also has the best the best fitted combination of sensitivity (69\%) and specificity (74\%) rates.

\begin{tabular}{|l|l|l|l|l|l|}
$\begin{array}{l}\text { Risk } \\
\text { factor }\end{array}$ & AUC & $\begin{array}{l}\text { P } \\
\text { Value }\end{array}$ & Cut-off & $\begin{array}{l}\text { Sensitivity } \\
(\%)\end{array}$ & $\begin{array}{l}\text { Specificity } \\
(\%)\end{array}$ \\
\hline AP $[\mathrm{mm}]$ & $.729(.58-.878)$ & .002 & 7.5 & $69.2 \%$ & $73.5 \%$ \\
\hline $\mathbf{C C}[\mathrm{mm}]$ & $.66(.512-.808)$ & .071 & 4.5 & $46.2 \%$ & $80.1 \%$ \\
\hline $\mathbf{V}\left[\mathrm{mm}^{3}\right]$ & $.686(.564-.808)$ & .055 & 123.3 & $100 \%$ & $36.8 \%$ \\
\hline
\end{tabular}


FIGURES:

Figure 1: Flowchart of patient inclusion process and image analysis. $\mathrm{T} 1 \mathrm{WI}=\mathrm{T} 1$-weighted imaging, $\mathrm{T} 2 \mathrm{WI}=$ T2-weighted imaging, ROC analysis $=$ Receiver operating characteristic analysis

Figure 2: Logistic regression analysis showing advantage of the AP dimension over other geometric criteria determining the appropriateness of using contrast in pituitary MRIs.

The forest plot of the odds ratios (OR) of lack of correct diagnosis of pituitary lesions in a conventional MRI examination (common pre- and post-contrast sequences), which includes the following risk factors: anteroposterior pituitary dimension (AP), cranio-caudal dimension (CC), transverse dimension (TR) and gland volume (V).

The forest plot based on the logistic regression analysis clearly shows that only AP dimension, which does not cross the line of no difference (1.0 value), is statistically significant in contrast to the other dimensions (CC, TR) and gland volume (V).

Figure 3: Antero-posterior (AP) dimension in the ROC analysis of chance for incorrect diagnosis of pituitary lesions in a conventional MRI examination in children with growth or puberty disorders.

The Receiver Operating Characteristic (ROC) analysis shows that the antero-posterior (AP) dimension is a statistically significant (Area Under Curve [AUC] lower CI 95\% >50\%) predictor of the appropriateness of contrast administration in the diagnosis of pituitary focal lesions in children with growth or puberty disorders (GPD). Classification of patients based on the AP cut-off $=7.5 \mathrm{~mm}$ indicates the proper post-contrast diagnosis in $(\mathrm{AUC} \sim 73 \%=) 3$ out of 4 patients. What is more, ROC analysis of the AP dimension testifies the best fitted combination of sensitivity (69\%) and specificity (74\%) rates, which reveals the high ability of this predictor to use gadolinium-based contrast agent (GBCA) correctly when required, and the proper omission of GBCA administration when unnecessary.

Figure 4: Cranio-caudal dimension in the ROC analysis of chance for incorrect diagnosis of pituitary lesions in a conventional MRI examination in children with growth or puberty disorders. 
The Receiver Operating Characteristic (ROC) analysis shows that the cranio-caudal (CC) dimension is a statistically significant (Area Under Curve [AUC] lower CI 95\% > 50\%) predictor of the appropriateness of contrast administration in the diagnosis of pituitary focal lesions in our research group. The CC cut-off $=4.5$ $\mathrm{mm}$ indicates the proper contrast diagnostics in (AUC $66 \%=) 2$ out of 3 patients. What is more, ROC analysis of the CC dimension testifies the lower sensitivity rate $=46 \%$ than specificity $=80 \%$, which indicates smaller capability of this predictor to use gadolinium-based contrast agent (GBCA) correctly when required.

Figure 5: Volume in the ROC analysis of chance for incorrect diagnosis of pituitary lesions in a conventional MRI examination in children with growth or puberty disorders.

The Receiver Operating Characteristic (ROC) analysis shows that also pituitary volume (V) is a statistically significant (Area Under Curve [AUC] lower CI 95\% >50\%) predictor of the appropriateness of contrast administration in the diagnosis of pituitary focal lesions in our research group. ROC analysis of the gland volume testifies the lower specificity rate $(37 \%)$ than sensitivity $(100 \%)$ and the volume cut-off $=123 \mathrm{~mm}^{3}$ indicates the proper contrast diagnostics in (AUC ) $69 \%$ of patients.

Figure 6. The practical approach for assessment of the pituitary MRI in children with growth or puberty disorders before hormonal therapy.

\section{Compliance with Ethical Standards:}

Conflict of Interest: The author (Marta Michali-Stolarska, Andrzej Tukiendorf, Jagoda JackówNowicka, Anna Zacharzewska-Gondek, Joanna Chrzanowska, Joanna Bladowska) declares that he/ she has no conflict of interest

Ethical approval: All procedures performed in studies involving human participants were in accordance with the ethical standards of the institutional and/or national research committee and with the 1964 Helsinki declaration and its later amendments or comparable ethical standards.

Approval for this research was given by the Commission of Ethics at the Wrockaw Medical University (number of permission: 117/2019). Informed consent was obtained from each patient to participate in this study. 
Financial support statement: Wroclaw Medical University Grant SUB.C270.21.020.

Ethical approval: This article does not contain any studies with animals performed by any of the authors.

Informed consent: Informed consent was obtained from all individual participants included in the study. 


\section{Figures}

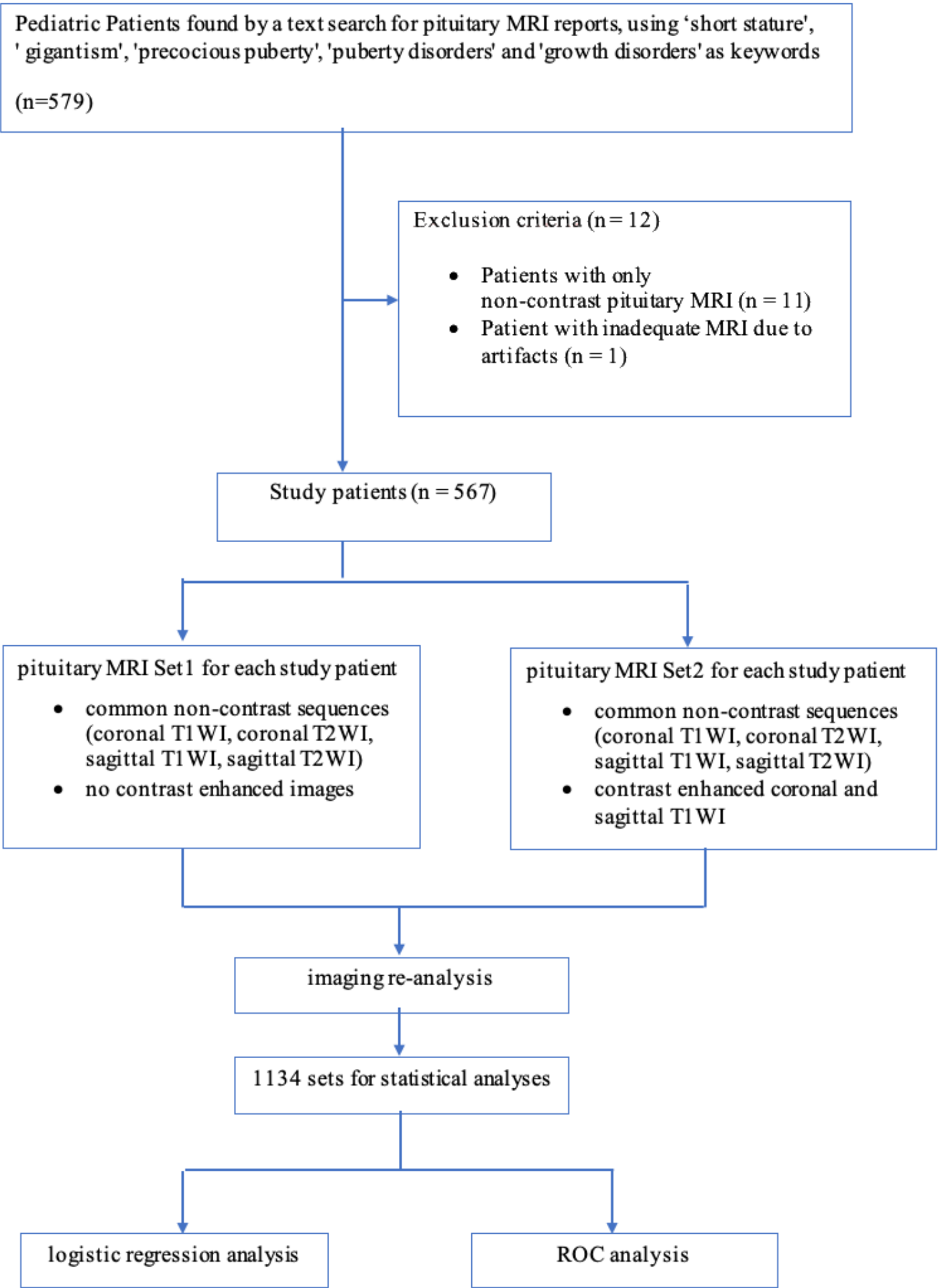

\section{Figure 1}

Flowchart of patient inclusion process and image analysis. T1WI = T1-weighted imaging, $\mathrm{T} 2 \mathrm{WI}=\mathrm{T} 2-$ weighted imaging, ROC analysis = Receiver operating characteristic analysis 


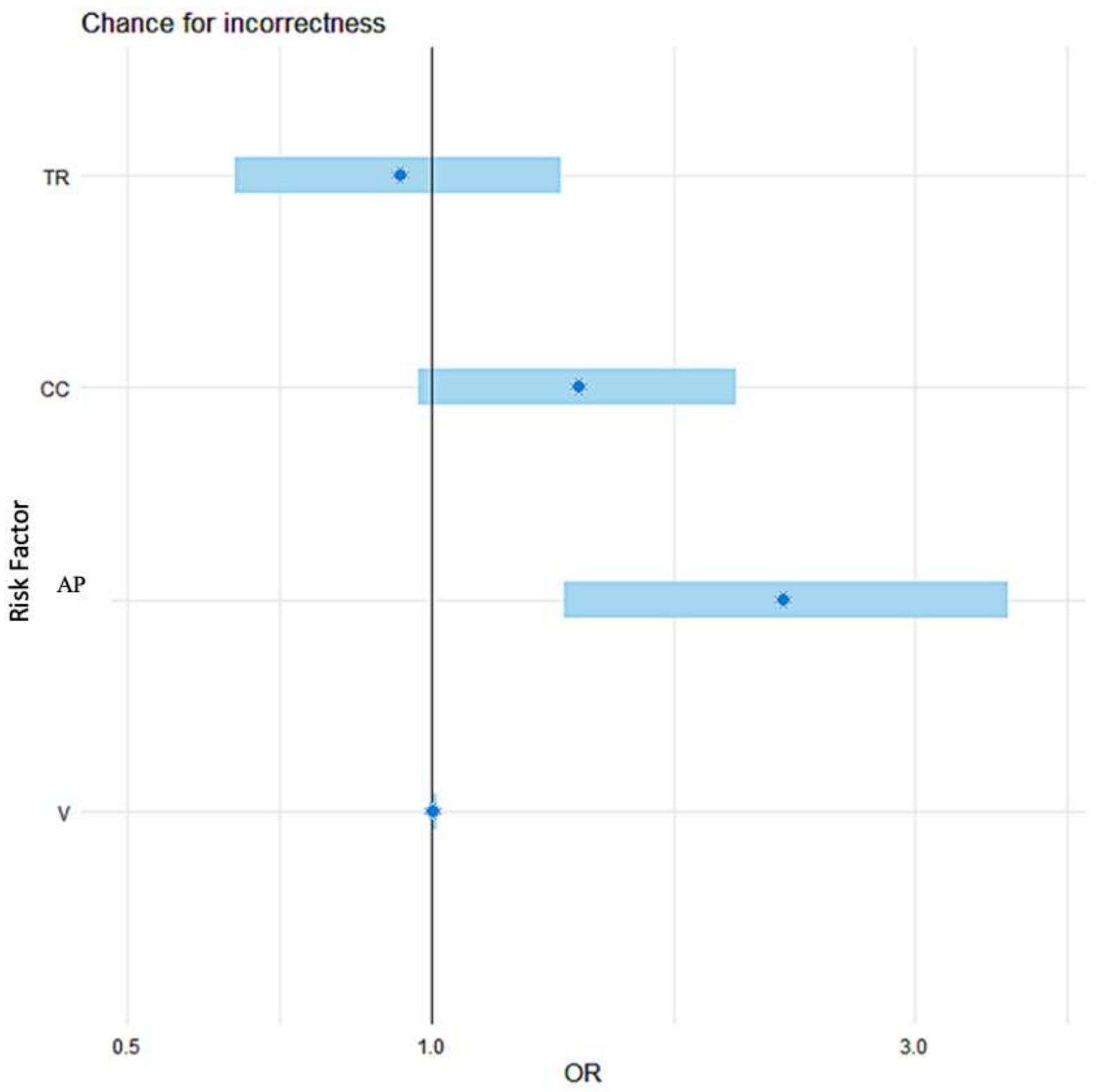

Figure 2

Logistic regression analysis showing advantage of the AP dimension over other geometric criteria determining the appropriateness of using contrast in pituitary MRIs. The forest plot of the odds ratios (OR) of lack of correct diagnosis of pituitary lesions in a conventional MRI examination (common preand post-contrast sequences), which includes the following risk factors: anteroposterior pituitary dimension (AP), cranio-caudal dimension (CC), transverse dimension (TR) and gland volume (V). The 
forest plot based on the logistic regression analysis clearly shows that only AP dimension, which does not cross the line of no difference ( 1.0 value), is statistically significant in contrast to the other dimensions (CC, TR) and gland volume (V).

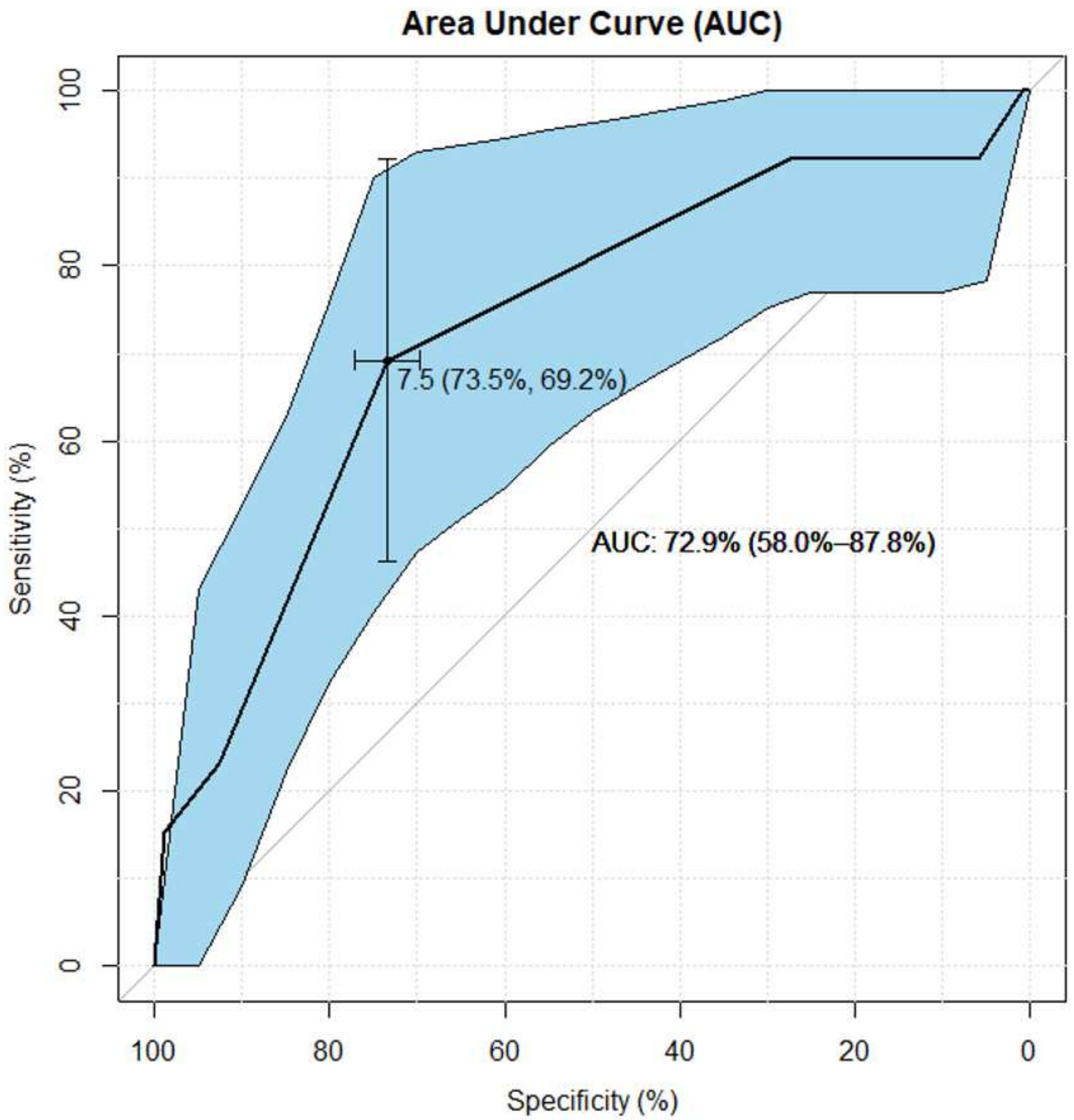

Figure 3

Antero-posterior (AP) dimension in the ROC analysis of chance for incorrect diagnosis of pituitary lesions in a conventional MRI examination in children with growth or puberty disorders. The Receiver Operating Characteristic (ROC) analysis shows that the antero-posterior (AP) dimension is a statistically significant 
(Area Under Curve [AUC] lower $\mathrm{Cl} 95 \%>50 \%$ ) predictor of the appropriateness of contrast administration in the diagnosis of pituitary focal lesions in children with growth or puberty disorders (GPD).

Classification of patients based on the AP cut-off $=7.5 \mathrm{~mm}$ indicates the proper post-contrast diagnosis in (AUC 73\% =) 3 out of 4 patients. What is more, ROC analysis of the AP dimension testifies the best fitted combination of sensitivity $(69 \%)$ and specificity $(74 \%)$ rates, which reveals the high ability of this predictor to use gadolinium-based contrast agent (GBCA) correctly when required, and the proper omission of GBCA administration when unnecessary.

Area Under Curve (AUC)

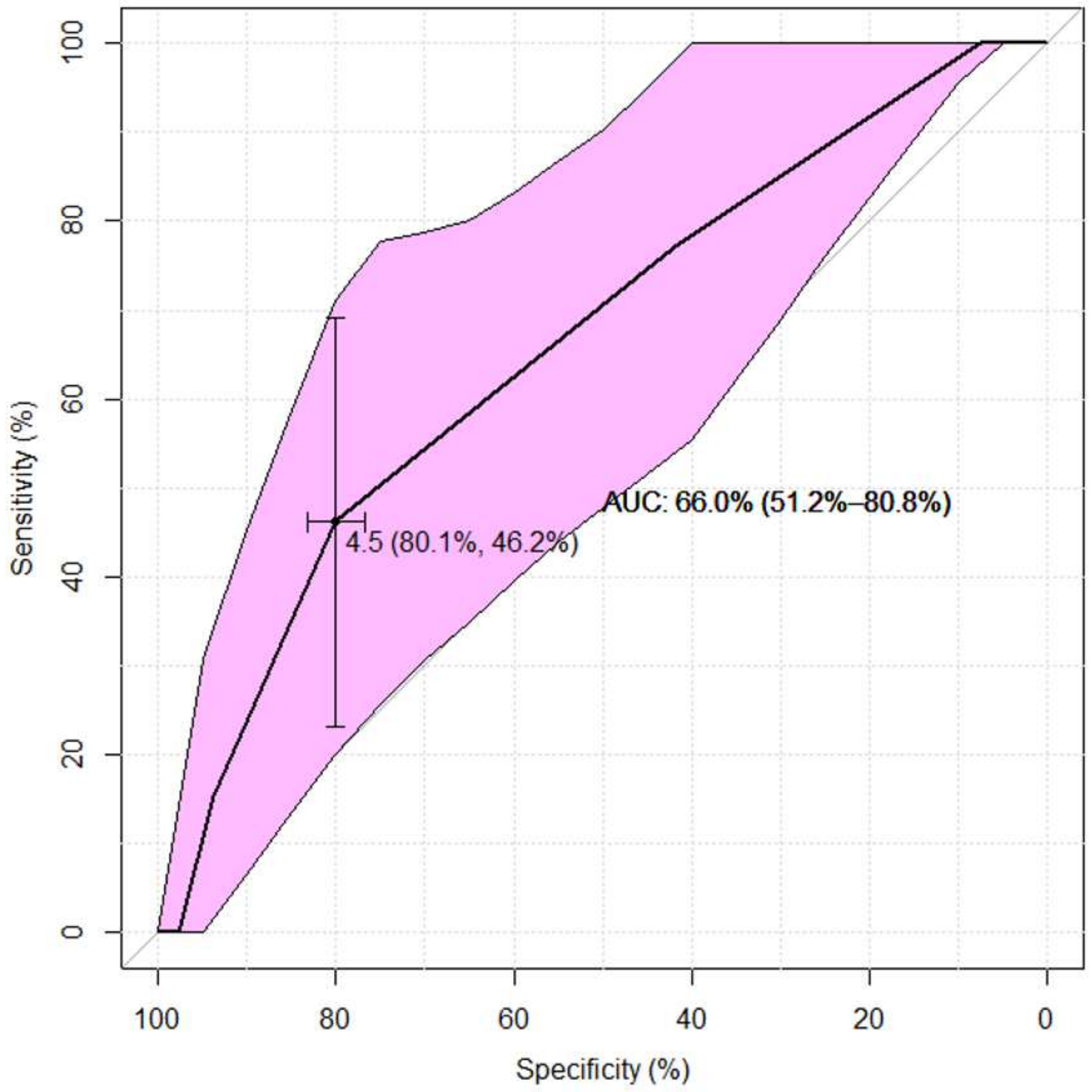

Figure 4 
Cranio-caudal dimension in the ROC analysis of chance for incorrect diagnosis of pituitary lesions in a conventional MRI examination in children with growth or puberty disorders. The Receiver Operating Characteristic (ROC) analysis shows that the cranio-caudal (CC) dimension is a statistically significant (Area Under Curve [AUC] lower $\mathrm{Cl} 95 \%>50 \%$ ) predictor of the appropriateness of contrast administration in the diagnosis of pituitary focal lesions in our research group. The CC cut-off $=4.5 \mathrm{~mm}$ indicates the proper contrast diagnostics in (AUC 66\% =) 2 out of 3 patients. What is more, ROC analysis of the CC dimension testifies the lower sensitivity rate $=46 \%$ than specificity $=80 \%$, which indicates smaller capability of this predictor to use gadolinium-based contrast agent (GBCA) correctly when required.

Area Under Curve (AUC)

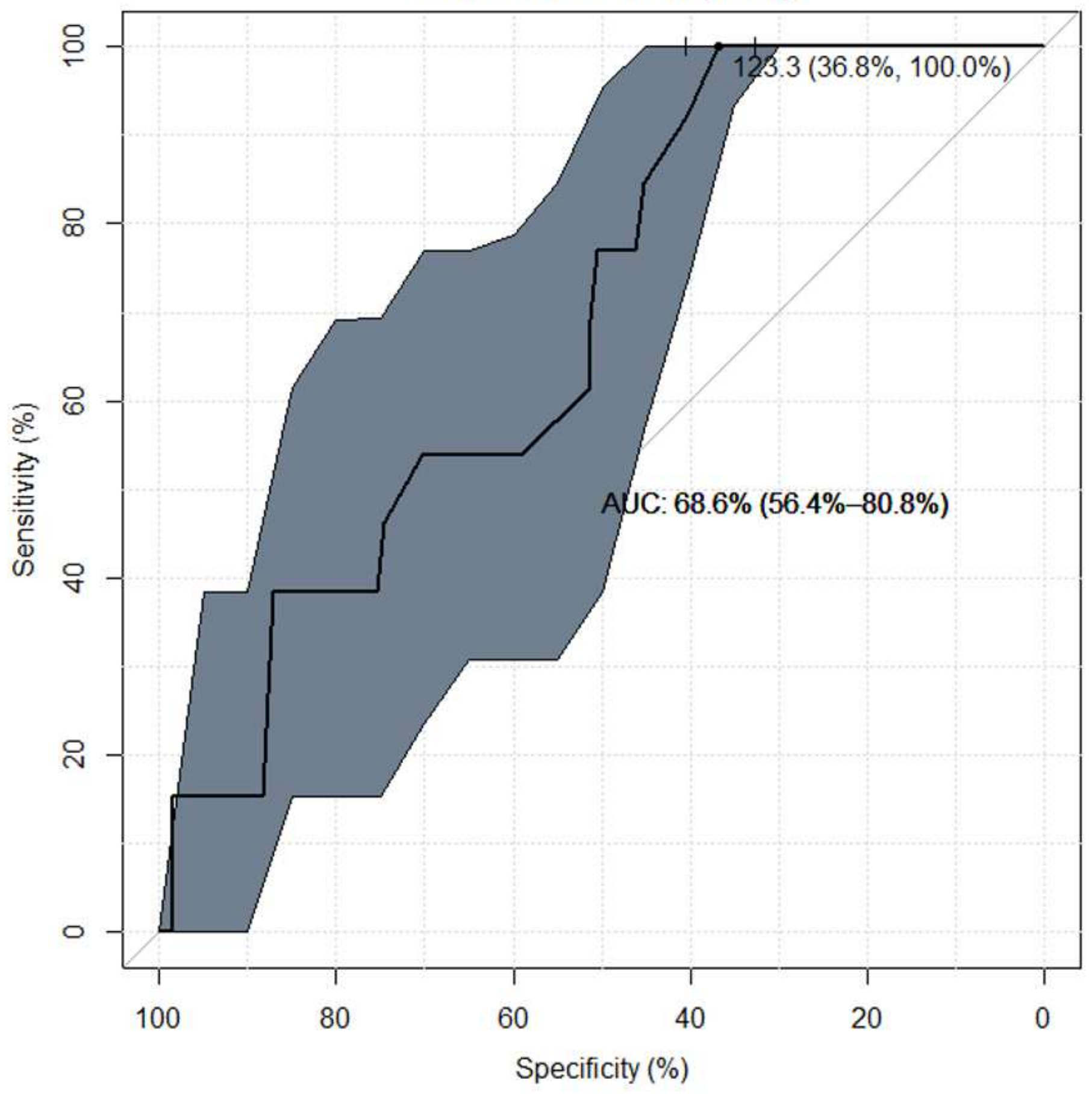




\section{Figure 5}

Volume in the ROC analysis of chance for incorrect diagnosis of pituitary lesions in a conventional MRI examination in children with growth or puberty disorders. The Receiver Operating Characteristic (ROC) analysis shows that also pituitary volume $(\mathrm{V})$ is a statistically significant (Area Under Curve [AUC] lower $\mathrm{Cl}$ $95 \%>50 \%$ ) predictor of the appropriateness of contrast administration in the diagnosis of pituitary focal lesions in our research group. ROC analysis of the gland volume testifies the lower specificity rate (37\%) than sensitivity $(100 \%)$ and the volume cut-off $=123 \mathrm{~mm} 3$ indicates the proper contrast diagnostics in (AUC) $69 \%$ of patients. 


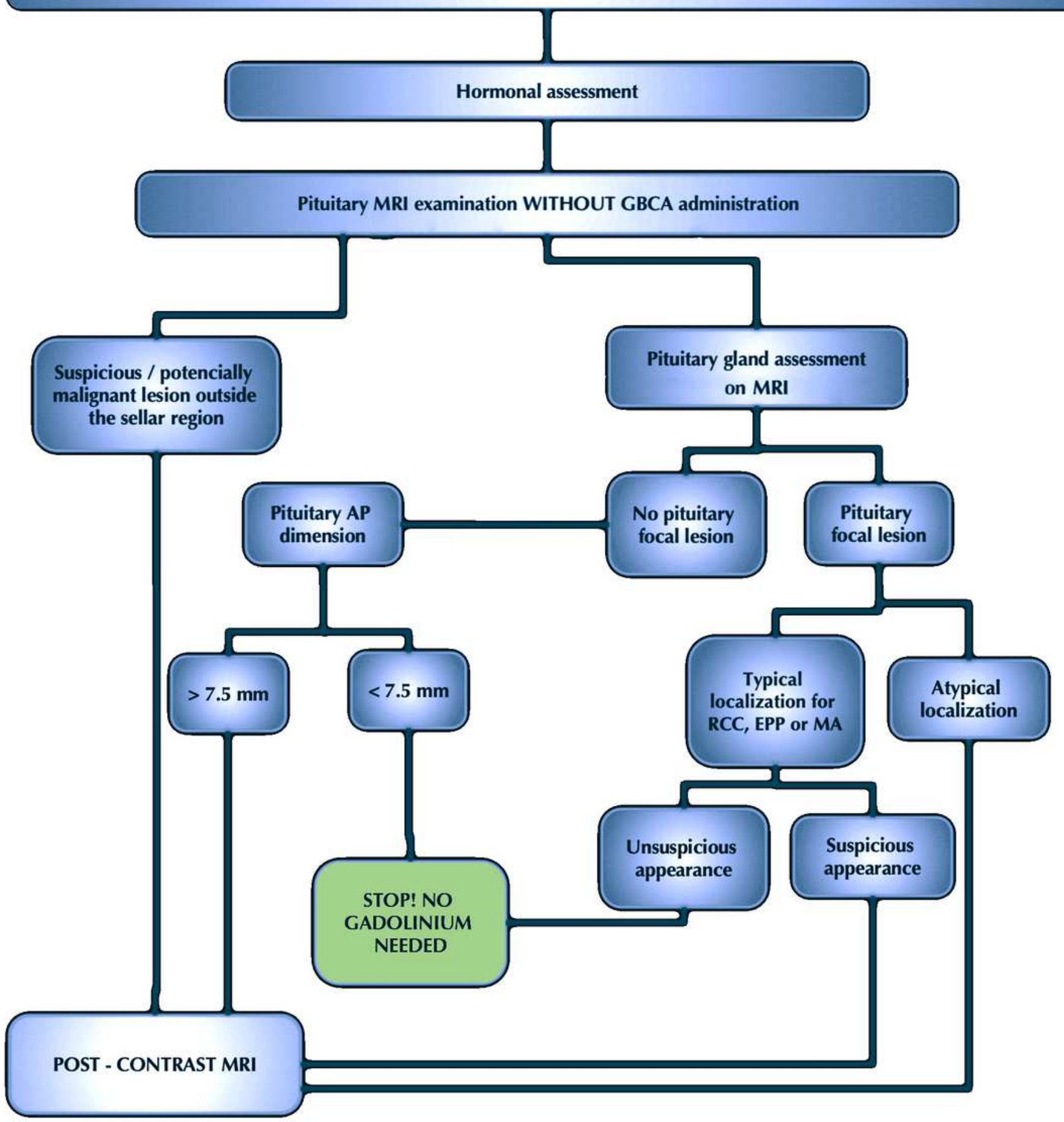

Figure 6

The practical approach for assessment of the pituitary MRI in children with growth or puberty disorders before hormonal therapy. 